\title{
Lymph Fistulas after Inguinal Lymph Node Dissection-Assessment of Risk Factors and Future Treatment Strategies
}

\author{
Nils Habbe*, Alexander Reinisch, Wolf Otto Bechstein, Sabine Hannes \\ Department of General and Visceral Surgery, University Hospital Frankfurt, Frankfurt, Germany \\ Email: "nils.habbe@kgu.de
}

Received 22 March 2014; revised 16 April 2014; accepted 23 April 2014

Copyright (C) 2014 by authors and Scientific Research Publishing Inc. This work is licensed under the Creative Commons Attribution International License (CC BY). http://creativecommons.org/licenses/by/4.0/

(c) (i) Open Access

\begin{abstract}
Background: Inguinal lymph node dissections (ILND) have been commonly associated with postoperative complications such as lymphedema, lymph fistula and wound infections. The reported complication rates after ILND range from $14 \%-77 \%$, and a rationale for these large differences is the use of a variety of different definitions of complications. Patients and Methods: Files of patients that underwent inguinal lymph node dissection for lymph node metastases at the Department of General and Visceral Surgery of the University Hospital Frankfurt between February 2005 and March 2012 were retrospectively reviewed. Results: 47 patients $(65 \%)$ developed a lymphatic fistula in the course of the operation, of which 6 patients $(8 \%)$ underwent reoperations. Surgical side infections (SSI) were seen in 15 patients (21\%), all of them harbouring fistulas. Patients presenting with a BMI $\geq 30 \mathrm{~kg} / \mathrm{m}^{2}$ did not develop more fistulas than those with a BMI $<30 \mathrm{~kg} / \mathrm{m}^{2}(\mathrm{p}=$ 0.30). Patients who suffered from a lymph fistula had significantly more visits in the outpatient clinic ( $p=<0.0001)$. There was no difference in the complication rate if the procedure was performed by a resident under supervision or a senior surgeon $(p=0.79)$. Conclusion: In this study, we demonstrated that lymph fistulas are to date a common complication after ILND and lead to reoperations and significantly more outpatient visits. The number of lymph nodes affected and resected, and prior sentinel lymph node dissection, was not associated with a higher complication rate.
\end{abstract}

\section{Keywords}

Lymph Fistula, Inguinal Lymph Node, Lymphadenectomy, Wound Infection

\footnotetext{
${ }^{*}$ Corresponding author.
} 


\section{Introduction}

Radical lymphadenectomy is the standard of care in melanoma patients with biopsy-proven or palpable lymph node metastases, and represents also a therapeutic approach in patients with lymph node metastases from other malignant diseases, even for debulking reasons [1]-[4].

Inguinal lymph node dissection (ILND) has been commonly associated with postoperative complications such as lymphedema, lymph fistula and wound infections leading towards a prolonged hospital stay, more visits in the outpatient clinic and thus delayed return to work and a reduced quality of life. The reported complication rates after ILND range from $14 \%-77 \%$, and a rationale for these large differences is the use of a variety of different definitions of complications. The lymph fistula, defined as a persisting discharge of lymph fluid over a drain, especially represents a therapeutic challenge in patients after ILND [5]-[8].

Several studies have addressed the issue of postoperative complications after ILND and determined predictors and associated factors of these complications. However, the development of lymph fistulas has not been sufficiently elaborated [9].

In this study, we retrospectively examined the incidence and factors associated with the development of lymph fistulas after ILND with a special focus on items that have not been analysed so far, for example the influence of residents on the development of fistulas.

\section{Patients and Methods}

We retrospectively reviewed records of all patients that underwent inguinal lymph node dissection for lymph node metastases at the Department of General and Visceral Surgery of the University Hospital Frankfurt between February 2005 and March 2012. The study has prior been approved by the Internal Review Board.

The preoperative diagnosis of inguinal lymph node metastases was formulated in all patients on the basis of follow-up examinations and imaging procedures according to the guidelines of the respected malignancy. The decision for radical lymphadenectomy in each case was made after thorough discussion in an interdisciplinary tumor board.

Clinical data, especially prior lymphadenectomies, number of resected lymph nodes, number of lymph node metastases and details of each operation, were collected.

The preoperative desinfection was performed in a standardized procedure using six Aseptoderm ${ }^{\circledR}$ (Dr. Schumacher GmbH, Germany) soaked swaps to wash the groin region, sterile draping but without using an incise drape after desinfection. Inguinal lymphadenectomy was performed using a vertical incision starting $2 \mathrm{~cm}$ superior of the inguinal ligament lateral of the vascular bundle. The superior boundary of the dissection was the anterior superior iliac spine (superior lateral) and the pubic tubercle (medial), the inferior, medial and lateral boundaries are the adductor longus and sartorius muscles. All lymphatic tissue within this triangle was dissected, sacrificing the saphenous vein in most of the cases. The femoral artery and vein were skeletonized over the anterior 180 degree of the surface. Furthermore, the tissue superior of the inguinal ligament within the medial and lateral boundaries was resected of the external oblique fascia. A 14 Charriere suction drain was placed in all cases and was removed if the output was $<30 \mathrm{ml} / 24 \mathrm{~h}$. All specimens were examined by an experienced pathologist.

Lymph fistula was defined as persisting flow of lymph fluid $>30 \mathrm{ml} /$ day for more than 3 days after the procedure or a significant leakage of lymphatic fluid for more than 5 days after the procedure, regardless of the exact amount [10].

The Mann-Whitney test, Fisher's exact test and $t$-test were used to evaluate the relationships between clinical features. A p-value $<0.05$ was considered statistically significant. Statistics were calculated using MedCalc ${ }^{\circledR}$ version 13.0.4. (MedCalc Software, Belgium).

\section{Results}

\subsection{Patients' Characteristics}

Seventy-two patients (34 female, 38 male) underwent inguinal lymphadenectomy between 2005 and 2012 at the Department of General and Visceral Surgery of the University Hospital Frankfurt. The median age at operation was 63 years (range: 33 - 83 years). The underlying malignancy was malignant melanoma in 61 patients, rectal cancer in 4 patients and 7 patients presented with inguinal lymph node metastases of a variety of other cancers 
(Table 1). The median body mass index (BMI) of the patients was $25.5 \mathrm{~kg} / \mathrm{m}^{2}$ (women: $25 \mathrm{~kg} / \mathrm{m}^{2}$; range: 16 - 37 $\mathrm{kg} / \mathrm{m}^{2}$; men: $25.5 \mathrm{~kg} / \mathrm{m}^{2}$; range: $21-37 \mathrm{~kg} / \mathrm{m}^{2} ; \mathrm{p}=0.56$ ) and did not differ significantly between the sexes.

Chemotherapy was administered in 13 (18\%) patients prior to surgery. The time between last chemotherapy treatment was 265 days (range: 13 - 1963 days).

\subsection{Surgical Aspects and Outcome}

The median operation time was 91 min (range: 35 - $275 \mathrm{~min}$ ). Of these operations, 42 (58\%) were performed by senior surgeons, whereas 30 (42\%) were performed by residents under supervision by a senior surgeon. There was no significant difference in operation time if the operation was done by a resident under supervision or by the senior surgeon herself/himself (residents: median $103.5 \mathrm{~min}$ (range: 38 - $195 \mathrm{~min}$ ) vs. seniors: 80 min (range: 35 - $275 \mathrm{~min}) ; \mathrm{p}=0.08)$.

The median number of resected lymph nodes in the specimen was 7 (range: 0 - 28). Fifty-one patients (71\%) revealed lymph node metastases in the final histopathology report, with a median of 1 (range: 0 - 12) lymph node metastasis per patient. Twenty-one patents (29\%) exhibited more than one metastasis. Thirty-six patients (50\%) underwent sentinel lymph node dissection (SLND) prior to the inguinal lymphadenectomy.

The median total hospital stay was 5 days (range: 3 - 34 days). Patients who developed a lymph fistula did not stay significantly longer in the hospital (median: 4 days; range: 1 - 24 days) compared to those without a fistula (median: 5 days; range: $1-8 ; \mathrm{p}=0.10$ ). Furthermore, patients who suffered from a lymph fistula had significantly more visits in the outpatient clinic (median: 4 ; range: 0 - 13 vs. median: 1 ; range: $0-6$; $p=<0.0001$ ).

\subsection{Complications and Associated Factors}

In total, 47 patients (65\%) developed a lymph fistula in the course of the operation, of which 6 patients (8\%) underwent reoperations. Surgical site infections (SSI) were seen in 15 patients (21\%), all of them harbouring fistulas. Out of these 15 patients with SSI, 5 (7\%) patients were treated with vacuum-assisted closure for wound conditioning after breakdown, whereas 8 (11\%) patients have been treated with antibiotics. There was no difference in the complication rate if the procedure was performed by a resident under supervision or the senior surgeon herself/himself $(\mathrm{p}=0.80)$.

The gender of the patients did not have an influence on the development of a fistula $(p=1.00)$. The BMI of patients with fistulas did not significantly differ from the BMI of patients with fistulas (median: $26 \mathrm{~kg} / \mathrm{m}^{2}$ (range: $16-37 \mathrm{~kg} / \mathrm{m}^{2}$ ) vs. $25 \mathrm{~kg} / \mathrm{m}^{2}$ (range: $20-36 \mathrm{~kg} / \mathrm{m}^{2}$ ); $\mathrm{p}=0.50$ ). Patients with a BMI $\geq 25 \mathrm{~kg} / \mathrm{m}^{2}$ did not reveal a significantly higher rate of fistulas compared to patients with lower BMI $(\mathrm{p}=0.46)$. Interestingly, patients presenting with a BMI $\geq 30 \mathrm{~kg} / \mathrm{m}^{2}$ did also not develop more fistulas than those with a BMI $<30 \mathrm{~kg} / \mathrm{m}^{2}(\mathrm{p}=0.30)$.

Patients who underwent SLND prior to the inguinal lymphadenectomy did not reveal significantly more lymphatic fistulas compared to patients without any surgery before $(\mathrm{p}=0.62)$. Patients who revealed more than 1 lymph node metastases in the final histopathology report did not have a higher fistula rate compared to patients with only 1 or without lymph node metastases $(\mathrm{p}=0.62)$ (Table 2).

\section{Discussion}

In this retrospective study of patients undergoing ILND for lymph node metastases, $65 \%$ of patients developed a lymph fistula, accompanied by a surgical site infection in $21 \%$ of all patients. In the cohort presented here, obesity (BMI $\geq 30$ ) had no influence on the complication rate. Furthermore, the number of affected lymph nodes was not associated with the occurrence of lymph fistulas.

Patients with lymph fistulas had not been significantly longer hospitalized, but revealed a significantly higher number of outpatient visits. Interestingly, there was no influence on the complication rate if the procedure was done by a resident under supervision or by the senior surgeon herself/himself.

The incidence of wound complications after ILND is significantly higher compared to lymph node dissections in other anatomical regions or other operations (1\% - 5\%).

Several explanations have been proposed to address this striking difference, including greater lymphatic flow, larger dissection area, thin skin flaps, density and type of the inguinal flora and difficulties in maintaining hygiene in this area, especially in obese patients [5] [11].

The incidence of postoperative complications after ILND resembles the complication rates reported by other 
Table 1. Patients' characteristics.

\begin{tabular}{|c|c|}
\hline Characteristic & Total $(n=72)$ \\
\hline \multicolumn{2}{|l|}{ Gender } \\
\hline Male & 38 \\
\hline Female & 34 \\
\hline Median age [years] & 63 \\
\hline (range) & $33-83$ \\
\hline Median BMI $\left[\mathrm{kg} / \mathrm{m}^{2}\right]$ & 25.5 \\
\hline (range) & $(16-37)$ \\
\hline \multicolumn{2}{|l|}{ BMI $\left[\mathrm{kg} / \mathrm{m}^{2}\right]$} \\
\hline$<25$ & 31 \\
\hline $25-30$ & 31 \\
\hline$>30$ & 10 \\
\hline Median Outpatient visits & 4 \\
\hline (range) & $(0-13)$ \\
\hline Median Hospital stay [days] & 5 \\
\hline (range) & $(3-34)$ \\
\hline \multicolumn{2}{|l|}{ Clarke's Level in MM patients } \\
\hline $\mathbf{I}$ & 0 \\
\hline II & 2 \\
\hline III & 8 \\
\hline IV & 41 \\
\hline $\mathbf{V}$ & 10 \\
\hline \multicolumn{2}{|l|}{ No. Lymph nodes involved* } \\
\hline $\mathbf{0}$ & 21 \\
\hline 1 & 30 \\
\hline $2-3$ & 9 \\
\hline$\geq 4$ & 12 \\
\hline Median Operation time [min] (range) & $91(35-275)$ \\
\hline Senior surgeon & $103.5(38-195)$ \\
\hline Residents & $80(35-275)$ \\
\hline
\end{tabular}

MM = malignant melanoma; ${ }^{*}$ Sentinel excluded.

Table 2. Factors associated with postoperative lymph fistulas.

\begin{tabular}{|c|c|c|c|c|}
\hline Factor & $\begin{array}{c}\text { Total } \\
(n=72)\end{array}$ & $\begin{array}{c}\text { No Fistula } \\
(n=25)\end{array}$ & $\begin{array}{c}\text { Fistula } \\
(n=47)\end{array}$ & p-Value \\
\hline BMI & & & & 0.46 \\
\hline$<25$ & 30 & 12 & 18 & \\
\hline$\geq 25$ & 42 & 13 & 29 & \\
\hline BMI & & & & 0.30 \\
\hline$<30$ & 61 & 23 & 38 & \\
\hline$\geq 30$ & 11 & 2 & 9 & \\
\hline Gender & & & & 1.00 \\
\hline Male & 38 & 12 & 26 & \\
\hline Female & 34 & 13 & 21 & \\
\hline Lymph node metastases & & & & 1.00 \\
\hline$\leq 1$ & 51 & 18 & 33 & \\
\hline$>1$ & 21 & 7 & 14 & \\
\hline Surgical experience & & & & 0.80 \\
\hline Resident & 30 & 11 & 19 & \\
\hline Senior surgeon & 42 & 14 & 28 & \\
\hline SLND before ILND & & & & 0.62 \\
\hline Yes & 36 & 11 & 25 & \\
\hline No & 36 & 14 & 22 & \\
\hline
\end{tabular}


groups. In the cohort reported by Chang and colleagues, $77 \%$ of all patients developed wound complications after ILND [5]. Similar results were presented by Serpell et al., with $71 \%$ of patients suffering from wound complications [11]. The incidence of complications reported by Coit and colleagues with $64 \%$ of patients revealing complications was similar to the rate presented in our study [12]. Interestingly, only $14 \%$ of all patients reported by Glarner et al. had developed wound complication after ILND [8]. The differences in the numbers of the reported complications in the published literature are likely based on the different definitions of wound complications employed in these reports. In addition, it is well established that obesity increases the risk for wound complications, which might serve as one reason for the lower wound complication rate in the cohort presented by Tonouchi et al. with a BMI of $22.5 \mathrm{~kg} / \mathrm{m}^{2}$ when compared to the cohort presented by Chang and colleagues, harbouring a BMI of $30.8 \mathrm{~kg} / \mathrm{m}^{2}$ [5] [13]. Mohr et al. also reported a significant association between a BMI around $30 \mathrm{~kg} / \mathrm{m}^{2}$ and lymph fistulas [9].

We could not demonstrate a significantly higher rate of wound complications in patients with a BMI $\geq 30$ $\mathrm{kg} / \mathrm{m}^{2}$ undergoing ILND, probably due to the small number of obese patients in our cohort $(\mathrm{n}=10)$.

In the cohort presented in our study, $42 \%$ of all ILND were performed by residents under the supervision of an experienced senior surgeon. The complication rate was not higher in the "operation by resident" group compared to the senior surgeon group, whereas the operation time was longer but did not reach significance. We have recently demonstrated similar findings in ileostomy reversals, backing our findings that procedures performed by residents do not have elevated rates of complications, only longer operation times [14].

It is a well-established fact that postoperative wound complications lead towards longer hospital stays, more readmissions or outpatient visits and an increase in overall costs [15] [16]. Chang and colleagues addressed this issue and estimated the cost in patients undergoing ILND with or without postoperative wound complications, revealing significantly increased costs for inpatient and outpatient treatment in patients with complications. In our study, we did not compare the costs for patients with or without lymph fistulas after ILND, but patients with fistulas revealed significantly more outpatient visits, which does not only lead towards increased costs, but also to a delayed return to work.

Several modification of the surgical technique have been described to date, addressing these high wound complication rates in patients undergoing ILND or vascular surgery of the groin. Giovannaci and colleagues revealed a significantly lower rate of complications in 224 patients after femoral artery surgery, either treated with a conventional closure or randomized to a group treated with fibrin glue [17]. The power of this study is limited, as lymph node dissections and vascular surgery in the groin region differ significantly in their impact on the lymphatic system. The difference in the surgical impact on the lymphatic system of either vascular surgery or radical lymph node dissections offers an explanation for the findings of Neuss et al. in their randomized trial on 58 patients undergoing iliacal and inguinal lymph node dissection for melanoma. In this study, patients receiving fibrin glue did not reveal lower wound complication rates [18].

Similar results have been reported on the use of collagen sealant patches (e.g. TachoSil ${ }^{\circledR}$ ) in the backdrop of lymph node dissections, in their single center trial on 70 patients undergoing axillary or ilio-inguinal lymph node dissection, Di Monte et al. reported a significantly higher percentage of drainage-free patients on day 21 after surgery in the TachoSil ${ }^{\circledR}$ group [19]. This study has several limitations. First, patients with a BMI $>30 \mathrm{~kg} / \mathrm{m}^{2}$ have been excluded, which is a group that has been identified for having an increased risk for the development of wound complications and fistulas in the aforementioned publications. Second, axillary and ilio-inguinal lymph node dissection differ significantly in the postoperative complication rate itself and thus cannot be pooled.

Besides fibrin glue and collagen patches, the use of other electrothermal sealing devices has been analysed in the context of lymph node dissections and postoperative lymph fistulas. Cortadellas and colleagues reported a significantly shorter operation time and fewer days of suction drain in 100 patients undergoing axillary dissection in their randomized clinical study using LigaSure ${ }^{\mathrm{TM}}$ [20]. The use of argon plasma coagulation in a single case of bilateral inguinal lymph node dissection revealed decreased lymph secretion rates in the area treated by argon plasma coagulation [21].

In summary, in depth randomized controlled multicenter studies addressing surgical techniques helping to prevent lymph fistulas and wound complications after ILND are not available to date.

\section{Conclusions}

In this study, we demonstrated that lymph fistulas are to date a common complication after ILND and lead to 
reoperations, significantly more outpatient visits and thus to a consumption of healthcare resources. Radical surgery, i.e. the number of lymph nodes affected and resected, and prior sentinel lymph node dissection, was not associated with a higher complication rate. Although the operation time was longer, procedures performed by residents were not associated with a higher complication rate.

In the backdrop of the current literature, this study once more demonstrates the need for randomized trials addressing surgical solutions for the high complication rates in inguinal lymph node dissections.

\section{Conflict of Interests}

The authors declare that there is no conflict of interests regarding the publication of this paper.

\section{References}

[1] Spillane, A.J., Pasquali, S., Haydu, L.E. and Thompson, J.F. (2014) Patterns of Recurrence and Survival after Lymphadenectomy in Melanoma Patients: Clarifying the Effects of Timing of Surgery and Lymph Node Tumor Burden. Annals of Surgical Oncology, 21, 292-299. http://dx.doi.org/10.1245/s10434-013-3253-6

[2] Spillane, A.J., Haydu, L., McMillan, W., Stretch, J.R. and Thompson, J.F. (2011) Quality Assurance Parameters and Predictors of Outcome for Ilioinguinal and Inguinal Dissection in a Contemporary Melanoma Patient Population. Annals of Surgical Oncology, 18, 2521-2528. http://dx.doi.org/10.1245/s10434-011-1755-7

[3] Hughes, T.M., A’Hern, R.P. and Thomas, J.M. (2000) Prognosis and Surgical Management of Patients with Palpable Inguinal Lymph Node Metastases from Melanoma. British Journal of Surgery, 87, 892-901.

http://dx.doi.org/10.1046/j.1365-2168.2000.01439.x

[4] Nathansohn, N., Schachter, J. and Gutman, H. (2005) Patterns of Recurrence in Patients with Melanoma after Radical Lymph Node Dissection. Archives of Surgery, 140, 1172-1177. http://dx.doi.org/10.1001/archsurg.140.12.1172

[5] Chang, S.B., Askew, R.L., Xing, Y., et al. (2010) Prospective Assessment of Postoperative Complications and Associated Costs Following Inguinal Lymph Node Dissection (ILND) in Melanoma Patients. Annals of Surgical Oncology, 17, 2764-2772. http://dx.doi.org/10.1245/s10434-010-1026-z

[6] de Vries, M., Vonkeman, W.G., van Ginkel, R.J., et al. (2006) Morbidity after Inguinal Sentinel Lymph Node Biopsy and Completion Lymph Node Dissection in Patients with Cutaneous Melanoma. Opean Journal of Surgical Oncology, 32, 785-789. http://dx.doi.org/10.1016/j.ejso.2006.05.003

[7] Ul-Mulk, J. and Hölmich, L.R. (2012) Lymph Node Dissection in Patients with Malignant Melanoma Is Associated with High Risk of Morbidity. Danish Medical Journal, 59, A4441.

[8] Glarner, C.E., Greenblatt, D.Y., Rettammel, R.J., Neuman, H.B. and Weber, S.M. (2013) Wound Complications after Inguinal Lymph Node Dissection for Melanoma: Is ACS NSQIP Adequate? Annals of Surgical Oncology, 20, 2049-2055. http://dx.doi.org/10.1245/s10434-012-2856-7

[9] Mohr, Z., Hirche, C., Gretschel, S. and Bembenek, A. (2011) Risk Factors for the Development of Lymphatic Fistula after Ilioinguinal Lymph Node Dissection before Isolated Limb Perfusion and Its Potential Clinical Relevance. Zentralblatt für Chirurgie, 136, 386-390. http://dx.doi.org/10.1055/s-0030-1262547

[10] Giovannacci, L., Renggli, J.C., Eugster, T., Stierli, P., Hess, P. and Gürke, L. (2001) Reduction of Groin Lymphatic Complications by Application of Fibrin Glue: Preliminary Results of a Randomized Study. Annals of Vascular Surgery, 15, 182-185. http://dx.doi.org/10.1007/s100160010049

[11] Serpell, J.W., Carne, P.W. and Bailey, M. (2003) Radical Lymph Node Dissection for Melanoma. ANZ Journal of Surgery, 73, 294-299. http://dx.doi.org/10.1046/j.1445-2197.2003.t01-1-02622.x

[12] Coit, D.G., Peters, M. and Brennan, M.F. (1991) A Prospective Randomized Trial of Perioperative Cefazolin Treatment in Axillary and Groin Dissection. Archives of Surgery, 126, 1366-1372. http://dx.doi.org/10.1001/archsurg.1991.01410350056009

[13] Tonouchi, H., Ohmori, Y., Kobayashi, M., Konishi, N., Tanaka, K., Mohri, Y., Mizutani, H. and Kusunoki, M. (2004) Operative Morbidity Associated with Groin Dissections. Surgery Today, 34, 413-418. http://dx.doi.org/10.1007/s00595-003-2738-5

[14] Habbe, N., Hannes, S., Liese, J., Woeste, G., Bechstein, W.O. and Strey, C. (2014) The Use of Purse-String Skin Closure in Loop Ileostomy Reversals Leads to Lower Wound Infection Rates-A Single High-Volume Centre Experience. International Journal of Colorectal Disease, Epub ahead of print. http://dx.doi.org/10.1007/s00384-013-1822-6

[15] Herwaldt, L.A., Cullen, J.J., Scholz, D., French, P., Zimmerman, M.B., Pfaller, M.A., Wenzel, R.P. and Perl, T.M. (2006) A Prospective Study of Outcomes, Healthcare Resource Utilization, and Costs Associated with Postoperative Nosocomial Infections. Infection Control and Hospital Epidemiology, 27, 1291-1298. 
http://dx.doi.org/10.1086/509827

[16] Fukuda, H., Morikane, K., Kuroki, M., Kawai, S., Hayashi, K., Ieiri, Y., Matsukawa, H., Okada, K., Sakamoto, F., Shinzato, T. and Taniguchi, S. (2012) Impact of Surgical Site Infections after Open and Laparoscopic Colon and Rectal Surgeries on Postoperative Resource Consumption. Infection, 40, 649-659.

http://dx.doi.org/10.1007/s15010-012-0317-7

[17] Giovannacci, L., Eugster, T., Stierli, P., Hess, P. and Gürke, L. (2002) Does Fibrin Glue Reduce Complications after Femoral Artery Surgery? A Randomised Trial. European Journal of Vascular and Endovascular Surgery, 24, 196-201. http://dx.doi.org/10.1053/ejvs.2002.1667

[18] Neuss, H., Raue, W., Koplin, G., Schwenk, W., Reetz, C. And Mall, J.W. (2009) A Prospective Randomized Trial: The Influence of Intraoperative Application of Fibrin Glue after Radical Inguinal/Iliacal Lymph Node Dissection on Postoperative Morbidity. Journal of Surgical Oncology, 35, 884-889. http://dx.doi.org/10.1016/j.ejso.2008.09.016

[19] Di Monta, G., Caracò, C., Crispo, A., Marone, U. and Mozzillo, N. (2012) Collagen Sealant Patch to Reduce Lymphatic Drainage after Lymph Node Dissection. World Journal of Surgical Oncology, 19, 10:275. http://dx.doi.org/10.1186/1477-7819-10-275

[20] Cortadellas, T., Córdoba, O., Espinosa-Bravo, M., Mendoza-Santin, C., Rodríguez-Fernández, J., Esgueva, A., Alvarez-Vinuesa, M., Rubio, I.T. and Xercavins, J. (2011) Electrothermal Bipolar Vessel Sealing System in Axillary Dissection: A Prospective Randomized Clinical Study. International Journal of Surgery, 9, 636-640. http://dx.doi.org/10.1016/j.ijsu.2011.08.002

[21] Madhuri, T.K., Tailor, A. and Butler-Manuel, S. (2011) Use of Neutral Plasma Coagulation in Groin Node Dissection for Vulvar Malignancy: A Novel Technique. Cancer Management and Research, 3, 253-255. http://dx.doi.org/10.2147/CMAR.S19467 